\title{
A connection-theoretic approach to the analysis of symmetries of nonholonomic field theories
}

\author{
J. Vankerschaver* \\ Dept. of Mathematical Physics and Astronomy, \\ Ghent University, Krijgslaan 281, \\ B-9000 Ghent, Belgium \\ E-mail: Joris.Vankerschaver@UGent.be
}

\begin{abstract}
For mechanical systems or classical field theories with nonholonomic constraints, the presence of symmetries does not immediately imply the existence of associated conservation laws, i.e. Noether's theorem is no longer valid and is replaced instead by an equation describing the evolution of the conserved quantity under the flow of the nonholonomic system. In this paper, we use the De Donder-Weyl formalism for nonholonomic field theories to derive this nonholonomic momentum equation.
\end{abstract}

Keywords: Nonholonomic constraints, classical field theories, symmetry

\section{Introduction}

Symmetries play a fundamental role in classical mechanics and field theory. This is epitomized in Noether's first theorem: every continuous symmetry gives rise to a conserved quantity. In the case where nonholonomic constraints are present, however, Noether's theorem is no longer valid, but the analysis of symmetries is still a very powerful tool, an observation which goes back at least to the work of Vierkandt. ${ }^{1}$

For nonholonomic mechanical systems with symmetry, instead of Noether's theorem, there exists an equation describing the evolution of the associated "conserved" quantities under the nonholonomic flow. This equation is know as the nonholonomic momentum equation and was derived by Bloch et al. ${ }^{2}$

*Present address: Control and Dynamical Systems, California Institute of Technology, 1200 E California Blvd, MC 107-81, Pasadena CA 91125 
for mechanical systems (see also the work of Cantrijn et $a l^{3}$ ).

In this paper, we derive a nonholonomic momentum equation for classical field theories with nonholonomic constraints (theorem 4.1). The main tool in our derivation is the nonholonomic De Donder-Weyl equation, which we recall in section 2 . In section 3 we introduce nonholonomic symmetries as sections of a certain bundle of Lie algebras. Section 4 is devoted to the proof of the nonholonomic momentum equation.

This paper is meant as a companion paper to Ref. 4, where proofs of some of the technical lemmata can be found, as well as a number of applications.

\section{Lagrangian first-order field theories}

\subsection{First-order jet bundles}

Throughout this paper, we will represent fields as sections of a given fibre bundle $\pi: Y \rightarrow X$, whose base space is an $(n+1)$-dimensional oriented manifold with volume form $\eta$. A typical coordinate system on $X$ is denoted as $\left(x^{\mu}\right), \mu=0, \ldots, n$, and is assumed to be adapted to the volume form in the sense that $\eta$ can locally be written as

$$
\eta=\mathrm{d}^{n+1} x:=\mathrm{d} x^{0} \wedge \cdots \wedge \mathrm{d} x^{n} .
$$

In addition, a typical coordinate system on $Y$ will be assumed to be adapted to the projection $\pi$, meaning that, if $\left(x^{\mu} ; y^{a}\right)$ are coordinates on $Y(a=$ $1, \ldots, m)$, then $\pi$ is locally given by $\pi\left(x^{\mu}, y^{a}\right)=\left(x^{\mu}\right)$.

The first jet bundle $J^{1} \pi$ is the manifold of equivalence classes of local sections of $\pi$, where two sections are said to be equivalent if their first-order Taylor expansions agree at a point. Elements of $J^{1} \pi$ are denoted as $j_{x}^{1} \phi$; there exists a projection $\pi_{1,0}: J^{1} \pi \rightarrow Y$ defined by $\pi_{1,0}\left(j_{x}^{1} \phi\right)=\phi(x)$. Furthermore, we define the projection $\pi_{1}: J^{1} \pi \rightarrow X$ as the composition $\pi \circ \pi_{1,0}$.

Recall that a connection on $\pi_{1}$ is a vector-valued one-form $\mathbf{h}$ on $J^{1} \pi$ such that $\mathbf{h}\rfloor \sigma=\sigma$ for every semi-basic one-form $\sigma$. Here, $\mathbf{h}\rfloor \sigma$ is the contraction of $\mathbf{h}$ with $\sigma$; see section A.1 for its definition. In coordinates, a connection can be represented as follows:

$$
\mathbf{h}=\mathrm{d} x^{\mu} \otimes\left(\frac{\partial}{\partial x^{\mu}}+\Gamma_{\mu}^{a} \frac{\partial}{\partial y^{a}}+\Gamma_{\mu \nu}^{a} \frac{\partial}{\partial y_{\nu}^{a}}\right),
$$

For more information about jet bundles and connections, see Ref. 5 . 


\section{The Cartan form}

For the purpose of this paper, a Lagrangian is a function $L$ on $J^{1} \pi$. Without going into any details, we mention here the existence of a distinguished $(n+1)$-form on $J^{1} \pi$, called the Cartan form, which is a central object in the geometric formulation of the field equations. If $L$ is a Lagrangian, then the associated Cartan form has the following coordinate expression:

$$
\Theta_{L}=\frac{\partial L}{\partial y_{\mu}^{a}}\left(\mathrm{~d} y^{a}-y_{\nu}^{a} \mathrm{~d} x^{\nu}\right) \wedge \mathrm{d}^{n} x_{\mu}+L \mathrm{~d}^{n+1} x .
$$

We define the Poincaré-Cartan form $\Omega_{L}$ as $-\mathrm{d} \Theta_{L}$.

\subsection{Nonholonomic constraints}

A nonholonomic field theory is determined by the specification of three elements (see also Refs. 4,6-8):

(1) a (regular) first-order Lagrangian $L: J^{1} \pi \rightarrow \mathbb{R}$;

(2) a constraint submanifold $\mathcal{C} \hookrightarrow J^{1} \pi$, such that the restriction of the projection $\left(\pi_{1,0}\right)_{\mid \mathcal{C}}$ defines a subbundle of $J^{1} \pi$;

(3) a bundle of reaction forces $F$, where the elements $\Phi$ of $F$ are $(n+1)$ forms defined along $\mathcal{C}$ which satisfy the following requirements:

(a) $\Phi$ is $n$-horizontal, i.e. $\Phi$ vanishes when contracted with any two $\pi_{1^{-}}$ vertical vector fields;

(b) $\Phi$ is 1 -contact, i.e. $\left(j^{1} \phi\right)^{*} \Phi=0$ for any section $\phi$ of $\pi$.

For the sake of simplicity, we assume that $\mathcal{C}$ is defined by the vanishing of $k$ functionally independent functions $\varphi^{\alpha}$ on $J^{1} \pi$, and that $F$ is globally generated by $l$ generators $\Phi^{\kappa}$ of the following form:

$$
\Phi^{\kappa}=A_{a}^{\kappa \mu}\left(\mathrm{d} y^{a}-y_{\nu}^{a} \mathrm{~d} x^{\nu}\right) \wedge \mathrm{d}^{n} x_{\mu} \quad(\kappa=1, \ldots, l) .
$$

In practice, the dimension $l$ of $F$ will be equal to the codimension $k$ of $\mathcal{C}$. There seems to be no a priori reason for supposing that $k=l$. In most cases, however, $F$ will be determined by $\mathcal{C}$ through application of the Chetaev principle (see Refs. 4,6) but this is not necessary for the present treatment. 


\subsection{The nonholonomic De Donder-Weyl equation}

The dynamics of a nonholonomic field theory is specified by the nonholonomic Euler-Lagrange equations, which are given below in coordinate form:

$$
\left[\frac{\partial L}{\partial y^{a}}-\frac{\mathrm{d}}{\mathrm{d} x^{\mu}} \frac{\partial L}{\partial y_{\mu}^{a}}\right]\left(j^{2} \phi\right)=\lambda_{\alpha \kappa} A_{a}^{\alpha \kappa}\left(j^{1} \phi\right) \quad \text { and } \quad \varphi^{\alpha}\left(j^{1} \phi\right)=0 .
$$

Here, $\lambda_{\alpha \kappa}$ are unknown Lagrange multipliers, to be determined from the constraints. Note that the terms on the right-hand side represent the reaction forces. A derivation of these equations can be found in Ref. 9 .

The dynamics of a field theory can also be approached through the socalled De Donder-Weyl equations, a set of algebraic equations specifying a connection $\mathbf{h}$ on $\pi_{1}$ with the following property: if $\psi$ is an integral section of $\mathbf{h}$, then $\psi$ is holonomic, i.e. there exists a section $\phi$ of $\pi$ such that $\psi=j^{1} \phi$, and in addition $\phi$ satisfies the Euler-Lagrange equations. In Ref. 6, the De Donder-Weyl equations were extended to the case of nonholonomic field theories and take the following form:

$$
i_{\mathbf{h}} \Omega_{L}-n \Omega_{L} \in \mathcal{I}(F) \text { and } \operatorname{Im} \mathbf{h} \subset T \mathcal{C},
$$

where $\mathcal{I}(F)$ is the ideal generated by $F$. These equations again specify a connection on $\pi_{1}$, whose integral sections are now the solutions of (2).

Remark 2.1. Throughout this paper, all Lagrangians are assumed to be regular, in the sense that the associated Hessian is nonsingular. If this is not the case, the correspondence between integral sections of the De Donder-Weyl equation and solutions of the Euler-Lagrange equations is not so straightforward.

\section{Nonholonomic symmetries}

Let $G$ be a Lie group acting on $\pi$ by bundle automorphisms, and assume that $G$ leaves invariant $L, \mathcal{C}$ and $F$, i.e. there exist smooth actions $\bar{\Phi}$ : $G \times Y \rightarrow Y$ and $\underline{\Phi}: G \times X \rightarrow X$ such that $\pi(\bar{\Phi}(g, y))=\underline{\Phi}(g, \pi(y))$ for all $g \in G$ and $y \in Y$. The Lie group $G$ then also acts on $J^{1} \pi$ by prolonged bundle automorphisms.

We consider first the bundle $\mathfrak{g}^{F}$ over $Y$, defined as follows: $\mathfrak{g}^{F}(y)$ is the linear subspace of $\mathfrak{g}$ consisting of all $\xi \in \mathfrak{g}$ such that

$$
\left.j^{1} \xi_{Y}(\gamma)\right\lrcorner F=0 \quad \text { for all } \gamma \in \mathcal{C} \cap \pi_{1,0}^{-1}(y),
$$


where $\xi_{Y}$ is the infinitesimal generator of the action corresponding to $\xi$, that is,

$$
\xi_{Y}(y)=\left.\frac{\mathrm{d}}{\mathrm{d} t}\right|_{t=0} \bar{\Phi}_{\exp (t \xi)}(y) .
$$

Next, we assume that the disjoint union of all $\mathfrak{g}^{F}(y)$, for all $y \in Y$ can be given the structure of a vector bundle over $Y$, which we denote by $\mathfrak{g}^{F}$. Note that to any section $\bar{\xi}$ of $\mathfrak{g}^{F}$, one can associate a vector field $\tilde{\xi}_{Y}$ on $Y$ according to the following prescription:

$$
\tilde{\xi}_{Y}(y)=[\bar{\xi}(y)]_{Y}(y) .
$$

Definition 3.1. A nonholonomic symmetry is a section $\bar{\xi}$ of $\mathfrak{g}^{F}$ such that the associated vector field $\tilde{\xi}_{Y}$ is $\pi$-projectable; i.e. there exists a vector field $\tilde{\xi}_{X}$ on $X$ such that $T \pi \circ \tilde{\xi}_{Y}=\tilde{\xi}_{X} \circ \pi$.

The following lemma, taken from Ref. 4, will be useful in the proof of the nonholonomic momentum equation.

Lemma 3.1. Let $\bar{\xi}$ be a section of $\mathfrak{g}^{F}$. For $y \in Y$, put $\xi:=\bar{\xi}(y)$ and consider any $\gamma \in \pi_{1,0}^{-1}(y) \cap \mathcal{C}$. Then there exists a $\pi_{1,0}$-vertical vector $v_{\gamma} \in$ $T_{\gamma} J^{1} \pi$ such that

$$
j^{1} \tilde{\xi}_{Y}(\gamma)=j^{1} \xi_{Y}(\gamma)+v_{\gamma}
$$

\section{The nonholonomic momentum equation}

Theorem 4.1. Let $\mathbf{h}$ be a solution of the nonholonomic De Donder-Weyl equation and consider an integral section $j^{1} \phi$ of $\mathbf{h}$. Then for any nonholonomic symmetry $\bar{\xi}$ the associated component of the momentum map $J_{\bar{\xi}}^{\text {n.h. }}$. satisfies the following nonholonomic momentum equation:

$$
\left(j^{1} \phi\right)^{*}\left(\mathrm{~d} J_{\bar{\xi}}^{\text {n.h. }}\right)=\left(j^{1} \phi\right)^{*}\left(\mathscr{L}_{j^{1}} \tilde{\xi}_{Y}(L \eta)\right) .
$$

Proof. Note first of all that if $j^{1} \phi$ is an integral section of $\mathbf{h}$, then the following holds:

$$
\left(j^{1} \phi\right)^{*}\left(\mathrm{~d} J_{\bar{\xi}}^{\text {n.h. }}\right)=\left(j^{1} \phi\right)^{*}\left(\mathrm{~d}_{\mathbf{h}} J_{\bar{\xi}}^{\text {n.h. }}\right) .
$$

Using lemma A.1, the $\mathbf{h}$-derivative of $J^{\text {n.h. }}$ on the right-hand side can be expanded as follows. 


$$
\begin{aligned}
\mathrm{d}_{\mathbf{h}} J_{\bar{\xi}}^{\text {n.h. }} & =\left(i_{\mathbf{h}} \mathrm{d}-\mathrm{d} i_{\mathbf{h}}\right) i_{j^{1} \tilde{\xi}_{Y}} \Theta_{L} \\
& =i_{\mathbf{h}} \mathscr{L}_{j^{1} \tilde{\xi}_{Y}} \Theta_{L}-i_{\mathbf{h}} i_{j^{1} \tilde{\xi}_{Y}} \mathrm{~d} \Theta_{L}-\mathrm{d} i_{\mathbf{h}} i_{j^{1}} \tilde{\xi}_{Y} \Theta_{L} \\
& =i_{\mathbf{h}} \mathscr{L}_{j^{1} \tilde{\xi}_{Y}} \Theta_{L}+i_{j^{1} \tilde{\xi}_{Y}} i_{\mathbf{h}} \Omega_{L}+i_{\mathbf{h}\left(j^{1} \tilde{\xi}_{Y}\right)} \mathrm{d} \Theta_{L}-\mathrm{d} i_{\mathbf{h}} i_{j^{1} \tilde{\xi}_{Y}} \Theta_{L} .
\end{aligned}
$$

Each of these terms will now be treated separately. Bear in mind that we will eventually pull back $\mathrm{d}_{\mathbf{h}} J_{\bar{\xi}}^{\text {n.h. }}$. to $X$ using a prolonged section $j^{1} \phi$ : this will get rid of all contact forms in the above expression, and for that purpose we introduce the following equivalence relation: we say that two forms $\alpha$ and $\beta$ on $J^{1} \pi$ are equivalent (denoted by $\alpha \simeq \beta$ ) if they agree up to a contact form, i.e. $\alpha \simeq \beta$ iff $\alpha=\beta+\theta$, where $\theta$ is contact. Note that this is equivalent to saying that $\left(j^{1} \phi\right)^{*} \alpha=\left(j^{1} \phi\right)^{*} \beta$ for all sections $\phi$ of $\pi$.

Term 1: $i_{\mathrm{h}} \mathscr{L}_{\boldsymbol{j}^{1} \tilde{\xi}_{Y}} \Theta_{L}$. Using lemma A.1, we have

$$
i_{\mathbf{h}} \mathscr{L}_{j^{1} \tilde{\xi}_{Y}} \Theta_{L}=\mathscr{L}_{j^{1} \tilde{\xi}_{Y}} i_{\mathbf{h}} \Theta_{L}-i_{\left[j^{1} \tilde{\xi}_{Y}, \mathbf{h}\right]} \Theta_{L} .
$$

To the first term, lemma A.2 can be applied. The second term vanishes, since $\left[j^{1} \tilde{\xi}_{Y}, \mathbf{h}\right]$ takes values in $V \pi_{1,0}$ (proposition 4.1) and $\Theta_{L}$ is semi-basic. In conclusion, term 1 is equal to

$$
i_{\mathbf{h}} \mathscr{L}_{j^{1} \tilde{\xi}_{Y}} \Theta_{L}=n \mathscr{L}_{j^{1} \tilde{\xi}_{Y}} \Theta_{L}+\mathscr{L}_{j^{1} \tilde{\xi}_{Y}}(L \eta) .
$$

Term 2: $i_{j^{1} \tilde{\xi}_{Y}} i_{\mathrm{h}} \Omega_{L}$. The nonholonomic De Donder-Weyl equations give us

$$
i_{j^{1} \tilde{\xi}_{Y}} i_{\mathbf{h}} \Omega_{L}=n i_{j^{1} \tilde{\xi}_{Y}} \Omega_{L}+i_{j^{1} \tilde{\xi}_{Y}} \zeta \simeq n i_{j^{1} \tilde{\xi}_{Y}} \Omega_{L},
$$

since $\zeta$ belongs to $\mathcal{I}(F)$, and hence can be written as $\zeta=\sum_{\alpha} \lambda^{\alpha} \wedge \Phi_{\alpha}$, for one-forms $\lambda^{\alpha}$. The contraction of $j^{1} \tilde{\xi}_{Y}$ with $\zeta$ then becomes

$$
i_{j^{1} \tilde{\xi}_{Y}} \zeta=\sum_{\alpha}\left(i_{j^{1} \tilde{\xi}_{Y}} \lambda^{\alpha}\right) \Phi_{\alpha}-\sum_{\alpha} \lambda^{\alpha} \wedge\left(i_{j^{1} \tilde{\xi}_{Y}} \Phi_{\alpha}\right)
$$

The first term is contact, and the second term vanishes since $j^{1} \tilde{\xi}_{Y}$ is admissible.

Term 3: $i_{\mathrm{h}\left(j^{1} \tilde{\xi}_{Y}\right)} \mathrm{d} \Theta_{L}$. In the case of vertical symmetries, as treated in Ref. 10, this term is automatically zero. In the general case, it is zero up to a contact form; the proof is rather technical. More precisely, we will show the following. If $j^{1} \phi$ is an integral section of the nonholonomic connection 
$\mathbf{h}$, then

$$
\left(j^{1} \phi\right)^{*}\left(i_{\mathbf{h}\left(j^{1} \tilde{\xi}_{Y}\right)} \mathrm{d} \Theta_{L}\right)=0
$$

Consider any such integral section $j^{1} \phi$, and fix a point $x$ in $X$. Put $y=\phi(x)$ and $\gamma=j_{x}^{1} \phi$ and define $\xi$ as $\bar{\xi}(y)$. Then lemma 3.1 allows us to conclude that

$$
\left.\left.\mathbf{h}\left(j^{1} \tilde{\xi}(\gamma)\right)\right\rfloor \mathrm{d} \Theta_{L}(\gamma)=\mathbf{h}\left(j^{1} \xi_{Y}(\gamma)\right)\right\rfloor \mathrm{d} \Theta_{L}(\gamma) .
$$

As $j^{1} \xi_{Y}$ is projectable (onto $\xi_{X}$ ), the last term is equal to $\left.T_{x} j^{1} \phi\left(\xi_{X}(x)\right)\right\lrcorner \mathrm{d} \Theta_{L}(\gamma)$.

For the pullback of term 3 under $j^{1} \phi$ we therefore have

$$
\left.\left.\left[\left(j^{1} \phi\right)^{*}\left(\mathbf{h}\left(j^{1} \tilde{\xi}_{Y}\right)\right\rfloor \mathrm{d} \Theta_{L}\right)\right]_{x}=\xi_{X}(x)\right\lrcorner\left[\left(j^{1} \phi\right)^{*} \mathrm{~d} \Theta_{L}\right]_{x},
$$

but the right-hand side is zero since $\mathrm{d} \Theta_{L}$ is an $(n+2)$-form pulled back to an $(n+1)$-dimensional space.

Term 4: $\mathrm{d} i_{\mathrm{h}} \boldsymbol{i}_{\boldsymbol{j}^{1} \tilde{\xi}_{Y}} \Theta_{L}$. Using lemma A.1, it follows that this term can be rewritten as

$$
\mathrm{d} i_{\mathbf{h}} i_{j^{1} \tilde{\xi}_{Y}} \Theta_{L}=\mathrm{d} i_{j^{1} \tilde{\xi}_{Y}} i_{\mathbf{h}} \Theta_{L}-\mathrm{d} i_{\mathbf{h}\left(j^{1} \tilde{\xi}_{Y}\right)} \Theta_{L}
$$

When considering the pullback of these terms under $j^{1} \phi$, a similar reasoning as the one leading to (5) shows that

$$
\left.\mathrm{d}\left(j^{1} \phi\right)^{*}\left(i_{\mathbf{h}\left(j^{1} \tilde{\xi}_{Y}\right)} \Theta_{L}\right)=\mathrm{d}\left(\xi_{X}\right\lrcorner\left(j^{1} \phi\right)^{*} \Theta_{L}\right),
$$

and this is in turn equal to $\left.\mathrm{d}\left(\xi_{X}\right\lrcorner\left(j^{1} \phi\right)^{*}(L \eta)\right)$, since $\Theta_{L} \simeq L \eta$. Using again lemma A.2, we conclude that

$$
\begin{aligned}
\left(j^{1} \phi\right)^{*}\left(\mathrm{~d} i_{\mathbf{h}} i_{j^{1} \tilde{\xi}_{Y}} \Theta_{L}\right) & =n\left(j^{1} \phi\right)^{*}\left(\mathrm{~d} i_{j^{1} \tilde{\xi}_{Y}} \Theta_{L}\right)+\left(j^{1} \phi\right)^{*}\left(\mathrm{~d} i_{j^{1} \tilde{\xi}_{Y}}(L \eta)\right. \\
& \left.+\mathrm{d}\left(\xi_{X}\right\rfloor\left(j^{1} \phi\right)^{*}(L \eta)\right)=n\left(j^{1} \phi\right)^{*}\left(\mathrm{~d} i_{j^{1} \tilde{\xi}_{Y}} \Theta_{L}\right) .
\end{aligned}
$$

Conclusion. Taking the pullback of $\mathrm{d}_{\mathbf{h}} J_{\bar{\xi}}^{\text {n.h. }}$ under an integral section $j^{1} \phi$ of $\mathbf{h}$, the foregoing allows us to write

$$
\left(j^{1} \phi\right)^{*}\left(\mathrm{~d}_{\mathbf{h}} J_{\bar{\xi}}^{\text {n.h. }}\right)=\left(j^{1} \phi\right)^{*}\left(\mathscr{L}_{j^{1} \tilde{\xi}_{Y}}(L \eta)\right) .
$$

This is the desired form of the nonholonomic momentum equation. 


\section{Acknowledgments}

I would like to thank D. Saunders and F. Cantrijn for their suggestions and comments.

I am a Postdoctoral Fellow from the Fund for Scientific Research - Flanders (FWO-Vlaanderen), and a Fulbright Research Scholar at the California Institute of Technology. Additional financial support from the Fonds Professor Wuytack is gratefully acknowledged.

\section{Appendix A. Technical lemmata}

The proof of the nonholonomic momentum equation uses a number of rather technical lemmata, which have been collected in this appendix.

\section{Appendix A.1. The Fröhlicher-Nijenhuis bracket}

The following lemma is a special case of lemma 8.6 in Ref. 11; a direct proof can be found in Ref. 10. Recall that the contraction $i_{\mathbf{h}} \alpha$ of a 1-1 tensor $\mathbf{h}$ with a $k$-form $\alpha$ is a $k$-form defined as follows:

$$
\left(i_{\mathbf{h}} \alpha\right)\left(v_{1}, \ldots, v_{k}\right)=\sum_{i=1}^{k}(-1)^{i+1} \alpha\left(\mathbf{h}\left(v_{i}\right), v_{1}, \ldots, \hat{v}_{i}, \ldots, v_{k}\right) .
$$

This $k$-form will sometimes be denoted as $\mathbf{h}\rfloor \alpha$.

Lemma A.1. Let $X$ be a vector field on $M$ and $\mathbf{h}$ a vector-valued one-form. Then, for any $k$-form $\alpha$ on $M$, the following holds:

(1) $i_{X} i_{\mathbf{h}} \alpha=i_{\mathbf{h}} i_{X} \alpha+i_{\mathbf{h}(X)} \alpha$;

(2) $i_{\mathbf{h}} \mathscr{L}_{X} \alpha=\mathscr{L}_{X} i_{\mathbf{h}} \alpha-i_{[X, \mathbf{h}]} \alpha$.

\section{Appendix A.2. Semi-holonomic connections}

Recall that a connection $\mathbf{h}$ on $\pi_{1}$ is said to be semi-holonomic if $i_{\mathbf{h}} \theta=0$ for all contact forms $\theta$ on $J^{1} \pi$. In coordinates, if $\mathbf{h}$ is locally represented as in (1), then $\mathbf{h}$ is semi-holonomic if $\Gamma_{\mu}^{a}=y_{\mu}^{a}$.

Lemma A.2. For each semi-holonomic connection $\Upsilon$ with horizontal pro- 
jector $\mathbf{h}$, the following holds:

$$
i_{\mathbf{h}} \Theta_{L}=n \Theta_{L}+L \eta
$$

Proof. This is Lemma 1 in Ref. 10.

Proposition 4.1. Let $X$ be a projectable vector field, and $\mathbf{h}$ a semiholonomic connection on $\pi_{1}$. Then the Fröhlicher-Nijenhuis bracket $\left[j^{1} X, \mathbf{h}\right]$ is a vector-valued one-form taking values in $V \pi_{1,0}$.

Proof. Let us write $X$ in coordinates as

$$
X=X^{\mu}(x) \frac{\partial}{\partial x^{\mu}}+X^{a}(x, y) \frac{\partial}{\partial y^{a}},
$$

and $\mathbf{h}$ as

$$
\mathbf{h}=\mathrm{d} x^{\mu} \otimes\left(\frac{\partial}{\partial x^{\mu}}+\Gamma_{\mu}^{a} \frac{\partial}{\partial y^{a}}+\Gamma_{\mu \nu}^{a} \frac{\partial}{\partial y_{\nu}^{a}}\right) .
$$

Note that $\Gamma_{\mu}^{a}$ is equal to $y_{\mu}^{a}$ since $\mathbf{h}$ is semi-holonomic.

The bracket $\left[j^{1} X, \mathbf{h}\right]$ is by definition just the Lie derivative $\mathscr{L}_{j^{1} X} \mathbf{h}$. Generally speaking, this vector-valued one-form takes values in $T J^{1} \pi$. A straightforward calculation shows that this form has the following expression:

$$
\begin{aligned}
\mathscr{L}_{j^{1} X} \mathbf{h} & =\left(\frac{\partial X^{\nu}}{\partial x^{\mu}}-\left(\frac{\partial}{\partial x^{\mu}}\right)^{H}\left(X^{\nu}\right)\right) \mathrm{d} x^{\mu} \otimes \frac{\partial}{\partial x^{\nu}} \\
& +\left(\Gamma_{\nu}^{a} \frac{\partial X^{\nu}}{\partial x^{\mu}}+j^{1} X\left(\Gamma_{\mu}^{a}\right)-\left(\frac{\partial}{\partial x^{\mu}}\right)^{H}\left(X^{a}\right)\right) \mathrm{d} x^{\mu} \otimes \frac{\partial}{\partial y^{a}} \\
& +(\ldots) \mathrm{d} x^{\mu} \otimes \frac{\partial}{\partial y_{\nu}^{a}}
\end{aligned}
$$

where we have used the following short-hand notation to denote the horizontal lift (with respect to the connection $\mathbf{h}$ ) of a vector field on $X$ :

$$
\left(\frac{\partial}{\partial x^{\mu}}\right)^{H}=\frac{\partial}{\partial x^{\mu}}+\Gamma_{\mu}^{a} \frac{\partial}{\partial y^{a}}+\Gamma_{\mu \nu}^{a} \frac{\partial}{\partial y_{\nu}^{a}}
$$

Expanding the terms between brackets now shows that the two first terms of (A.1) vanish, meaning that $\left[j^{1} X, \mathbf{h}\right]$ takes values in $V \pi_{1,0}$. 


\section{References}

1. A. Vierkandt, Monatsh. f. Math. 3, 31 (1892).

2. A. Bloch, P. Krishnaprasad, J. E. Marsden and R. Murray, Arch. Rat. Mech. Anal. 136, 21 (1996).

3. F. Cantrijn, M. de León, J. C. Marrero and D. Martín de Diego, J. Math. Phys. 40, 795 (1999).

4. J. Vankerschaver and D. Martín de Diego, Symmetry aspects of nonholonomic field theories. Preprint (2007).

5. D. J. Saunders, The Geometry of Jet Bundles, London Mathematical Society Lecture Note Series, Vol. 142 (Cambridge University Press, 1989).

6. J. Vankerschaver, F. Cantrijn, M. de León and D. Martín de Diego, Rep. Math. Phys. 56, 387 (2005).

7. O. Krupková, J. Diff. Eq. 220, 354 (2005).

8. O. Krupková and P. Volný, Lobachevskii J. Math. 23, 95 (2006).

9. E. Binz, M. de León, D. Martín de Diego and D. Socolescu, Rep. Math. Phys. 49, 151 (2002).

10. J. Vankerschaver, Int. J. Geom. Meth. Mod. Phys. 2, 1029 (2005).

11. I. Kolář, P. Michor and J. Slovák, Natural operations in differential geometry (Springer-Verlag, Berlin, 1993). 